\title{
Aitken Sequences and Generalized Fibonacci Numbers
}

\author{
By J. H. McCabe and G. M. Phillips
}

\begin{abstract}
Consider the sequence $\left(v_{n}\right)$ generated by $v_{n+1}=a v_{n}-b v_{n-1}, n \geqslant 2$, where $v_{1}=1$. $l_{2}=a$, with $a$ and $b$ real, of which the Fibonacci sequence is a special case. It is shown that if Aitken acceleration is used on the sequence $\left(x_{n}\right)$ defined by $x_{n}=v_{n+1} / v_{n}$, the resulting sequence is a subsequence of $\left(x_{n}\right)$. Second, if Newton's method and the secant method are used (with suitable starting values) to solve the equation $x^{2}-a x+b=0$, then the sequences obtained from both of those methods are also subsequences of the original sequence.
\end{abstract}

1. Introduction. Recently, the second author [5] showed that if Aitken acceleration is applied to the sequence of ratios of consecutive Fibonacci numbers, the resulting sequence is a subsequence of the original sequence. Specifically, let $u_{1}=u_{2}=1$ and $u_{n+1}=u_{n}+u_{n-1}, n \geqslant 2$. Define the sequence $\left(x_{n}\right)$ by

$$
x_{n}=u_{n+1} / u_{n}, \quad n=1,2, \ldots,
$$

and the Aitken sequence $\left(x_{n}^{*}\right)$ by

$$
x_{n}^{*}=\frac{x_{n+1} x_{n-1}-x_{n}^{2}}{x_{n+1}-2 x_{n}+x_{n-1}}, \quad n \geqslant 2 .
$$

Phillips [5] showed that

$$
x_{n}^{*}=x_{2 n}, \quad n \geqslant 2,
$$

and found that the repeated use of Aitken acceleration also gives subsequences of the original sequence (with repeated doubling of the suffix as above). This procedure will be stated more precisely in Section 4 .

The sequence $\left(x_{n}\right)$ converges linearly to $(1+\sqrt{5}) / 2$, the 'golden number', the positive root of the equation $x^{2}-x-1=0$. See Vorob'ev [6]. Phillips [5] also showed that when Newton's method and the secant method are applied to the equation $x^{2}-x-1=0$ with suitable starting values, the resulting sequences are again subsequences of $\left(x_{n}\right)$.

The purpose of this paper is to show that all of the above results generalize when the Fibonacci sequence is replaced by

$$
\begin{gathered}
v_{n+1}=a v_{n}-b v_{n-1}, \quad n \geqslant 2, \\
v_{1}=1, \quad v_{2}=a,
\end{gathered}
$$

where $a$ and $b$ are real numbers chosen so that no $v_{n}$ is zero. (Necessary and sufficient conditions on $a$ and $b$ are given in the next section.) Then, as before, we define $\left(x_{n}\right)$ by

$$
x_{n}=v_{n+1} / v_{n}
$$

Received July 10, 1984; revised January 22, 1985.

1980 Mathematics Subject Classification. Primary 65B05, 65H05, 41A21.

Key words and phrases. Fibonacci sequence, Aitken acceleration, Newton's method, secant method, Padé approximation, continued fraction. 
The original case is recovered by choosing $a=-b=1$. We note that another very simple case is that for which $a=2, b=1$, which yields $v_{n}=n$.

The generalization of the result (2) was prompted by a well-known relation between certain Padé approximants and Aitken acceleration [1, Section 3.1]. It is possible to justify the generalization of (2) by using continued fraction representations of the sequence of these Padé approximants. However, this leads to a presentation which is, to our taste, less satisfactory than the more direct method which we use here. Moreover, our direct method is compatible with the verification of our results in Section 5 on the Newton and secant methods.

2. The Recurrence Relation. In this section we obtain necessary and sufficient conditions on the real numbers $a$ and $b$ for the sequence (3) to have no zero members, so that $x_{n}$ is always defined by (4). Obviously, we require $a \neq 0$ and we will also avoid $b=0$, which gives a trivial case. (It is possible to allow $a$ and $b$ to be complex, but we will not pursue this.)

There are three cases to consider, depending on whether $x^{2}-a x+b=0$ has roots which are real and equal, real and unequal, or complex. If the roots are equal, we readily find that

$$
v_{n}=n(a / 2)^{n-1}
$$

and hence $v_{n} \neq 0$ for all $n$. Note that, in this case, the sequence $\left(x_{n}\right)$ converges to $a / 2$.

If the roots, say $\alpha$ and $\beta$, are unequal, we obtain

$$
v_{n}=\left(\alpha^{n}-\beta^{n}\right) /(\alpha-\beta) .
$$

Since $\alpha \neq \beta$ and $\alpha \neq-\beta$ (because $\alpha+\beta=a \neq 0$ ), we conclude that $v_{n} \neq 0$ for all $n$ when the roots are real and unequal. We also observe that in this case the sequence $\left(x_{n}\right)$ converges to the root of larger modulus.

The other possibility when the roots are unequal is that they are complex. In this case we have $\beta=\bar{\alpha}$. Let us write $\alpha=r e^{i \phi}$. Since $\alpha+\beta=a$ and $\alpha \beta=r^{2}=b$, we find that

$$
\cos \phi=\frac{1}{2} a / \sqrt{b}
$$

and, from (6),

$$
v_{n}=b^{\frac{1}{2}(n-1)} \sin n \phi / \sin \phi .
$$

In fact, we can write

$$
v_{n}=b^{\frac{1}{2}(n-1)} U_{n-1}(t),
$$

where $t=\frac{1}{2} a / \sqrt{b}$ and $U_{n-1}$ is the Chebyshev polynomial of the second kind, defined for $-1 \leqslant x \leqslant 1$ by

$$
U_{n-1}(x)=\sin n \theta / \sin \theta, \text { where } x=\cos \theta .
$$

These satisfy $U_{0}(x)=1, U_{1}(x)=2 x$ and the recurrence relation

$$
U_{n+1}(x)=2 x U_{n}(x)-U_{n-1}(x), \quad n \geqslant 1 .
$$

The zeros of $U_{n-1}(x)$ are easily seen to be $x=\cos (k \pi / n), 1 \leqslant k \leqslant n-1$. Thus, for all $v_{n}$ to be nonzero, we need to ensure that $t=\frac{1}{2} a / \sqrt{b}$ is not a zero of any of the polynomials $U_{n-1}(x)$. This entails that

$$
\cos ^{-1}\left(\frac{1}{2} a / \sqrt{b}\right) \neq k \pi / n,
$$


where $k$ and $n$ are integers such that $1 \leqslant k \leqslant n-1$. As an example of an inadmissible choice of $a$ and $b$ we cite the case $a=1, b=\frac{1}{3}$, for which we find that $v_{6}=0$. Note that, in the case of complex roots, the sequence $\left(x_{n}\right)$ has no limit.

3. Some Identities. We require the following three identities:

$$
\begin{aligned}
& v_{n+1} v_{m}-b v_{n} v_{m-1}=v_{n+m}, \\
& v_{n} v_{m+1}-v_{n+1} v_{m}=b^{m} v_{n-m}, \\
& v_{n}^{2}-v_{n+m} v_{n-m}=b^{n-m} v_{m}^{2} .
\end{aligned}
$$

The first identity is valid for $n \geqslant 1$ and $m \geqslant 2$ and the other two for all $n>m \geqslant 1$. The identity (9) is easily shown to be true for $m=2$ and all $n$ and also for $m=3$ and all $n$. The proof of (9) is completed by an induction argument, with induction on $m$. The same procedure serves to verify (10), except that we take 1 and 2 as the initial values of $m$. Thus, in verifying (9) and (10), all that we require besides induction is the recurrence relation (3) with its starting values.

To justify (11) we may proceed as follows. Let us write

$$
w_{n}=v_{n}^{2}-v_{n+1} v_{n-1}, \quad n \geqslant 2,
$$

and it is clear that $w_{2}=b$. We can show from (3) that $w_{n}-b w_{n-1}=0$ and hence that

$$
w_{n}=v_{n}^{2}-v_{n+1} v_{n-1}=b^{n-1} .
$$

This is (11) with $m=1$. For a general value of $m$ we now substitute for $v_{n+m}$ and $v_{n-m}$ in (11) from (9) and (10) respectively. The verification of (11) then follows after a little manipulation in which we need use only (3) and (12). For the special case of the Fibonacci sequence ((3) with $a=-b=1$ ), the identities (9) and (11) are quoted in Hoggatt [3].

It is interesting to observe that the sequence $\left(v_{n}\right)$, as defined by (3), can be extended so that $v_{n}$ is defined for all integers $n$, and not simply for positive integers. Thus we find that $v_{0}=0$ and

$$
v_{-n}=-b^{-n} v_{n}, \quad n>0 .
$$

Then the identity (10) is obtained from (9) on replacing $m$ by $-m$.

Before leaving these identities we note that it is also interesting to interpret them (and verify them) in terms of the explicit forms which we obtained earlier for $v_{n}$, where $v_{n}$ is written in one of the following ways, depending on the roots $\alpha$ and $\beta$ of the equation $x^{2}-a x+b=0$.

Equal roots: $\quad v_{n}=n(a / 2)^{n-1}$,

Complex roots: $\quad v_{n}=b^{(n-1) / 2} \sin n \phi / \sin \phi$,

$$
\text { where } \phi=\cos ^{-1}\left(\frac{1}{2} a / \sqrt{b}\right) \text {, }
$$

The identities (9), (10) and (11) are easily verified for $v_{n}$ given by (13), in which case we note that $b=\left(\frac{1}{2} a\right)^{2}$. We see that the exponential factor $(a / 2)^{n-1}$ which occurs in $v_{n}$ factors out and the identities behave as if $b=1$ and $v_{n}=n$. In this case the identities are concerned with the natural numbers. 
For $v_{n}$ given by (14), again the exponential factor $b^{(n-1) / 2}$ can be factored out. This time we have identities involving the sine function.

We are thus encouraged to look again at the form of $v_{n}$ in (15). There are different cases to consider depending on the signs of the roots of $\alpha$ and $\beta$ which, without loss of generality, we can consider satisfy $|\alpha|>|\beta|>0$. For example, if $\alpha>\beta>0$ we can rewrite (15) as

$$
v_{n}=b^{(n-1) / 2} \sinh n \lambda / \sinh \lambda, \quad \lambda=\cosh ^{-1}\left(\frac{1}{2} a / \sqrt{b}\right),
$$

and, in fact, the form (8) serves to describe both (7) and (16).

4. Aitken Acceleration. Here we show that

$$
\frac{x_{n+r} x_{n-r}-x_{n}^{2}}{x_{n+r}-2 x_{n}+x_{n-r}}=x_{2 n}, \quad n>r \geqslant 1 .
$$

Only the special case of (17) with $r=1$ is required to verify that Aitken acceleration on $\left(x_{n}\right)$ yields $x_{n}^{*}=x_{2 n}$. However, the full generality of (17) is needed to show what happens when we use repeated Aitken acceleration.

The numerator on the left of (17) becomes

$$
x_{n+r} x_{n-r}-x_{n}^{2}=\frac{v_{n+r+1} v_{n-r+1} v_{n}^{2}-v_{n+r} v_{n-r} v_{n+1}^{2}}{v_{n+r} v_{n-r} v_{n}^{2}} .
$$

The numerator on the right of (18) may be replaced by

$$
v_{n+1}^{2}\left(v_{n}^{2}-v_{n+r} v_{n-r}\right)-v_{n}^{2}\left(v_{n+1}^{2}-v_{n+r+1} v_{n-r+1}\right)
$$

and, on using (11) followed by (9), this last expression simplifies to give

$$
b^{n-r} v_{r}^{2} v_{2 n+1} \text {. }
$$

Thus the numerator on the left of (17) is

$$
x_{n+r} x_{n-r}-x_{n}^{2}=\frac{b^{n-r} v_{r}^{2} v_{2 n+1}}{v_{n+r} v_{n-r} v_{n}^{2}} .
$$

We now consider the denominator in the left of (17). First we obtain

$$
x_{n}-x_{n-r}=\frac{v_{n+1} v_{n-r}-v_{n-r+1} v_{n}}{v_{n} v_{n-r}}=\frac{-b^{n-r} v_{r}}{v_{n} v_{n-r}},
$$

on using (10). Hence we have

$$
x_{n+r}-2 x_{n}+x_{n-r}=\frac{b^{n-r} v_{r}\left(v_{n+r}-b^{r} v_{n-r}\right)}{v_{n+r} v_{n} v_{n-r}} .
$$

If we subtract (10) from (9), with $m=r$, and use (3), we obtain

$$
v_{n+r}-b^{r} v_{n-r}=v_{r}\left(v_{n+1}-b v_{n-1}\right)
$$

and so (20) becomes

$$
x_{n+r}-2 x_{n}+x_{n-r}=\frac{b^{n-r} v_{r}^{2}\left(v_{n+1}-b v_{n-1}\right)}{v_{n+r} v_{n} v_{n-r}} .
$$

Now from (9), with $m=n$,

$$
v_{n}\left(v_{n+1}-b v_{n-1}\right)=v_{2 n} .
$$

From this, (19) and (21) we obtain the required result (17). 
As we remarked above, (17) with $r=1$ shows that $x_{n}^{*}=x_{2 n}$. If we now write $x_{n}^{* *}$ to denote the result of applying Aitken acceleration to $x_{n-1}^{*}, x_{n}^{*}$ and $x_{n+1}^{*}$, we have

$$
x_{n}^{* *}=\frac{x_{n+1}^{*} x_{n-1}^{*}-\left(x_{n}^{*}\right)^{2}}{x_{n+1}^{*}-2 x_{n}^{*}+x_{n-1}^{*}}=\frac{x_{2 n+2} x_{2 n-2}-x_{2 n}^{2}}{x_{2 n+2}-2 x_{2 n}+x_{2 n-2}},
$$

from the result just proved. If we now use (17) with $r=2$ and $n$ replaced by $2 n$ we see that

$$
x_{n}^{* *}=x_{4 n} .
$$

We can obviously repeat Aitken acceleration as many times as we please and at each stage we obtain a subsequence of the original sequence. Each time we accelerate, the suffix is doubled.

5. Newton and Secant Methods. Consider the application of Newton's method to solve the equation $x^{2}-a x+b=0$. Given an initial approximant $t_{0}$, we compute a sequence $\left(t_{n}\right)$ from

$$
t_{n+1}=t_{n}-\frac{t_{n}^{2}-a t_{n}+b}{2 t_{n}-a}, \quad n \geqslant 0 .
$$

If $t_{n}=v_{k+1} / v_{k}$ for some value of $k$, we find that $t_{n+1}=v_{2 k+1} / v_{2 k}$. If we choose $t_{0}=v_{2} / v_{1}=a$ as the initial approximant, an induction argument shows that

$$
t_{n}=v_{2^{n}+1} / v_{2^{n}} \text {. }
$$

In the secant method we approximate to a root of the equation $f(x)=0$ as follows: we choose two initial approximants $t_{1}$ and $t_{2}$ and compute the sequence $\left(t_{n}\right)$ from

$$
t_{n+1}=t_{n}-\frac{f\left(t_{n}\right)\left(t_{n}-t_{n-1}\right)}{f\left(t_{n}\right)-f\left(t_{n-1}\right)}, \quad n \geqslant 2 .
$$

For $f(x) \equiv x^{2}-a x+b$ this gives

$$
t_{n+1}=\frac{t_{n} t_{n-1}-b}{t_{n}+t_{n-1}-a}, \quad n \geqslant 2 .
$$

If we choose $t_{1}=v_{p+1} / v_{p}$ and $t_{2}=v_{q+1} / v_{q}$, for some $p \neq q$, we find that $t_{3}=$ $v_{p+q+1} / v_{p+q}$. (This result, and the corresponding one described above for Newton's method, was obtained by Gill and Miller [2] for the case where the quadratic equation $x^{2}-a x-b=0$ has roots of unequal magnitude. This restriction is not necessary.) We see by induction that if we choose, as initial values, $t_{1}=a, t_{2}=a-$ $b / a$, the secant method gives

$$
t_{n}=v_{u_{n+1}+1} / v_{u_{n+1}},
$$

where $u_{n}$ is the $n$th Fibonacci number, the special case of $v_{n}$ for $a=-b=1$.

From the pedagogical point of view, the above results provide a very simple comparison of the Newton and secant methods. Such a comparison is usually achieved by asymptotic arguments involving the Fibonacci sequence. See, for example, Isaacson and Keller [4, pp. 99-101]. However, in this case the two methods yield subsequences of the same sequence $\left(x_{n}\right)$ which has first-order convergence (when it does converge). Thus we need only compare the suffices of the two 
sequences given by (23) and (24). To provide a fair comparison, we should replace $n$ by $n+2$ in (24) so that we use $n$ iterations of each process. We therefore compare the Fibonacci number $u_{n+3}$ with $2^{n}$. From (6) with $a=-b=1$ we deduce that

$$
u_{n+3} \sim \frac{1}{\sqrt{5}}\left(\frac{\sqrt{5}+1}{2}\right)^{n+3}=\left(1+\frac{2}{\sqrt{5}}\right) \cdot\left(\frac{\sqrt{5}+1}{2}\right)^{n} \text {. }
$$

We now extend this comparison to include the repeated use of Aitken acceleration. If, in principle, we compute the first $N+1$ members of the original sequence $\left(x_{n}\right)$ and then carry out as many Aitken accelerations as we can, how will the 'final number' compare with that obtained from $N$ iterations of the Newton or secant method? It is convenient to take $N=2 n$, and it is helpful to think of the Aitken accelerants set out in a table whose first column consists of the numbers $x_{1}, \ldots, x_{2 n+1}$. The second column contains the results of the first Aitken acceleration, namely $x_{2}^{*}, \ldots, x_{2 n}^{*}$. Successive columns contain the numbers obtained from each acceleration. Each column has two fewer members than the previous one. Thus we obtain a triangular array of numbers whose final number, obtained after $n$ accelerations, is $x_{(n+1) \cdot 2^{n}}$. As we have seen, $2 n$ iterations of Newton's method gives $x_{2^{2 n}}$.

The Mathematical Institute

University of St. Andrews

St. Andrews, Scotland KY16 9SS

1. G. A. Baker \& P. R. Graves-Morris, Padé Approximants, Part 1, Basic Theory, Vol. 13, Encyclopedia of Mathematics and its Applications, Addison-Wesley, Reading, Mass., 1981.

2. J. Gill \& G. Miller, “Newton's method and ratios of Fibonacci numbers," Fibonacci Quart., v. 19, 1981 , pp. 1-4.

3. V. E. Hoggatt, Fibonacci and Lucas Numbers, Houghton Mifflin, Boston, Mass., 1969

4. E. IsaAcson \& H. B. Keller, Analysis of Numerical Methods, Wiley, New York, 1966.

5. G. M. Phillips, “Aitken sequences and Fibonacci numbers," Amer. Math. Monthly, v. 91, 1984, pp. $354-357$.

6. N. N. Vorob'ev, Fibonacci Numbers, Popular Lectures in Mathematics Series, Vol. 2 (transl. from Russian), Pergamon Press, Oxford, 1961. 